\title{
Cluster effects on optical properties of glass-metal nanocomposites
}

\author{
G C DAS ${ }^{1}$ and D CHAKRAVORTY ${ }^{2}$ \\ 'Department of Metallurgical Engineering, Jadavpur University, Calcutta 700 032, India \\ ${ }^{2}$ Indian Association for the Cultivation of Science, Jadavpur, Calcutta 700032 , India \\ MS received 16 February 1989; revised 25 May 1989
}

\begin{abstract}
Optical absorption characteristics in a glass-metal nanocomposite system involving bismuth metal have been analysed using effective medium theories with a model incorporating single strand chains and $f c c$ clusters of metallic bismuth particles. The computed values show fair agreement with experimental data.
\end{abstract}

Keywords. Nanocomposites; optical absorption; effective medium theory; constrained optimization.

\section{Introduction}

Optical properties of dielectric-metal nanocomposites have attracted considerable attention in recent years (Granqvist and Hunderi 1980) because of their potential application in photothermal energy conversion devices. Nanocomposite films have so far been prepared either by gas evaporation (Granqvist and Buhrman 1976) or by r.f. sputtering (Craighead and Buhrman 1977). The optical absorption characteristics of these materials have been analysed by effective medium theories (Niklasson et al 1981) and show reasonable agreement with experimental data. Glass-metal nanocomposites have also been studied earlier (Doremus 1964; Kreibig 1974). Some recent investigations on nanocomposites involving bismuth metal and oxide glasses show interesting memory-switching behaviour (Chakravorty and Murthy 1975). This has been explained as arising due to the formation of a percolation path by the connectivity of stretched metal granules under the influence of an applied electric field (Das and Chakravorty 1980). The extremely low value of the volume fraction of the metal phase (typically in the range 0.03 to 0.12 ) implies that the interconnectivity is brought about by the bismuth particles which form a minor phase within the glass matrix. This is indicated also from the TEM microstructures (Das and Chakravorty 1982). Optical properties of these nanocomposites have been reported earlier (Das et al 1983) and the data analysed by various effective medium theories. In these analyses we have made the assumption that the bismuth metal phase consists of spheres only. On the basis of microstructural characteristics and the switching phenomenon exhibited by the bismuth-metal vanadium-phosphate glass nanocomposites as delineated above, we have reanalysed the optical data by assuming that the metal phase consists of spheres, single strand chains and $f c c$ clusters (Granqvist and Hunderi 1977). The agreement between the experimental values of the optical absorption coefficient and those computed as above is found to be much better than that reported earlier (Das et al 1983). The results are presented in this paper.

\section{Experimental}

The starting composition of the glass-metal nanocomposite chosen for the study 
is $80 \mathrm{~V}_{2} \mathrm{O}_{5}-15 \mathrm{P}_{2} \mathrm{O}_{5}-5 \mathrm{Bi}_{2} \mathrm{O}_{3}(\mathrm{~mol} \%)$. The details of sample preparation, optical absorption measurements and microstructural characterization have been reported earlier (Das et al 1983). Figure 1a is the transmission electron micrograph of the specimen used in the present investigation. The sample was subjected to reduction treatment at $200^{\circ} \mathrm{C}$ for $2 \mathrm{~h}$. The dark regions in this micrograph represent the bismuth-rich phase. The figure describes a two-dimensional projection of bismuth particles distributed in a three-dimensional nanocomposite. The volume fraction of the metal phase has been estimated by considering the thickness of the TEM sample (Das 1982). Figure 1b is the selected area diffraction pattern for the region described by figure 1a. The rings confirm the presence of metallic bismuth in the nanocomposites investigated.

\subsection{Calculations}

The simplest effective medium theory is the one developed by Maxwell-Garnett (MG) (1904). Polder and Van Santen (PVS) (1946) incorporated Onsager's reaction field to derive an improved effective medium permeability $\left(\bar{\varepsilon}^{\mathrm{MG}-\mathrm{PVS}}\right)$ for particles of ellipsoidal shape and is given by

$$
\bar{\varepsilon}^{\mathrm{MG}-\mathrm{PVS}}=\varepsilon_{m} \frac{1+(2 / 3) \sum_{j} f_{j} \delta_{j}}{1-(1 / 3) \sum_{j} f_{j} \delta_{j}}
$$

where $f_{j}$ is the fill factor of particles belonging to the $j$ th class such that $\sum_{j} f_{j}=f$, where $f$ is the fill factor, $\varepsilon_{m}$ is the dielectric permeability of the matrix. $\delta_{j}$ is related to polarizability of the particles and is given by (Granquist and Hunderi 1977)

$$
\delta_{j}=\frac{1}{3} \sum_{k=1}^{3}\left[\frac{\varepsilon_{j}-\bar{\varepsilon}^{\mathrm{MG}-\mathrm{PVS}}}{\overline{\bar{\varepsilon}} \overline{\mathrm{MG}-\mathrm{PVS}}+L_{k}\left(\varepsilon_{j}-\bar{\varepsilon} \overline{\mathrm{MG}-\mathrm{PVS}}\right)}\right],
$$

where $L_{k}$ are the depolarization factors of particles and $\varepsilon_{j}$ is the size-dependent dielectric permittivity. The frequency $(\omega)$ dependent optical absorption coefficient is given by (Granquist and Hunderi 1977)

$$
\alpha(\omega)^{\mathrm{MG}-\mathrm{PVs}}=(\omega / \mathrm{c})\left[\bar{\varepsilon}_{2} / \bar{\varepsilon}_{1}^{1}\right],
$$

where $\bar{\varepsilon}_{2}$ and $\bar{\varepsilon}_{1}$ are the imaginary and real parts of dielectric permittivity of (1).

For reasons given earlier, we assume that the bismuth phase contains $\xi$ fraction of spheres, $\zeta$ fraction of single strand chains and $\chi(=1-\xi-\zeta)$ fraction of $f c c$ clusters. The effect of these geometrical configurations can be incorporated in the MG-PVS theory by suitably replacing $L_{k}$ in (2). Our objective is to find the optimum combination of $\xi, \zeta$ and $\chi$ to get the best agreement with experimental results. It is evident that any two of these can be taken as the adjustable parameters in fitting the absorption spectra. So the objective function to be minimised is given by

$$
f(\mathbf{X})=\sum_{\omega=1}^{n}\left[\alpha(\omega)^{\mathrm{MG}-\mathrm{PVS}}-\alpha(\omega)^{\text {Expt. }}\right]^{2} .
$$




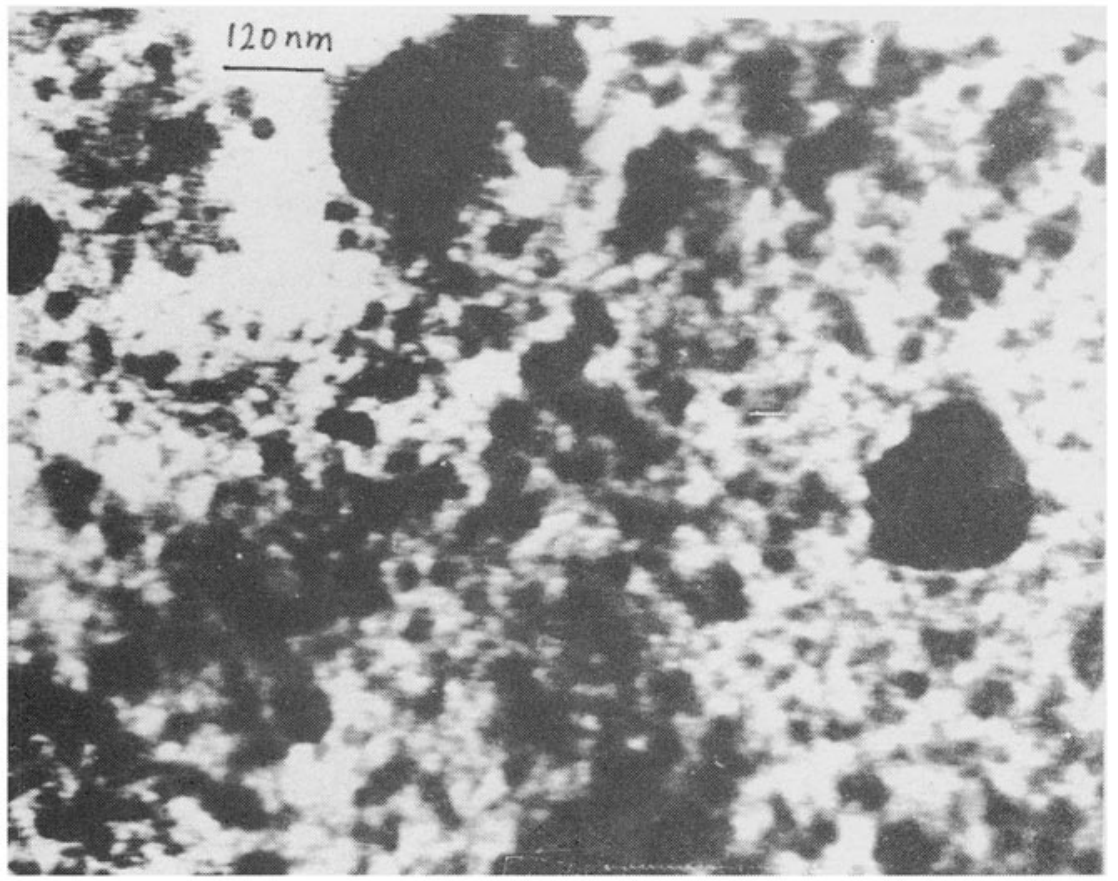

(a)

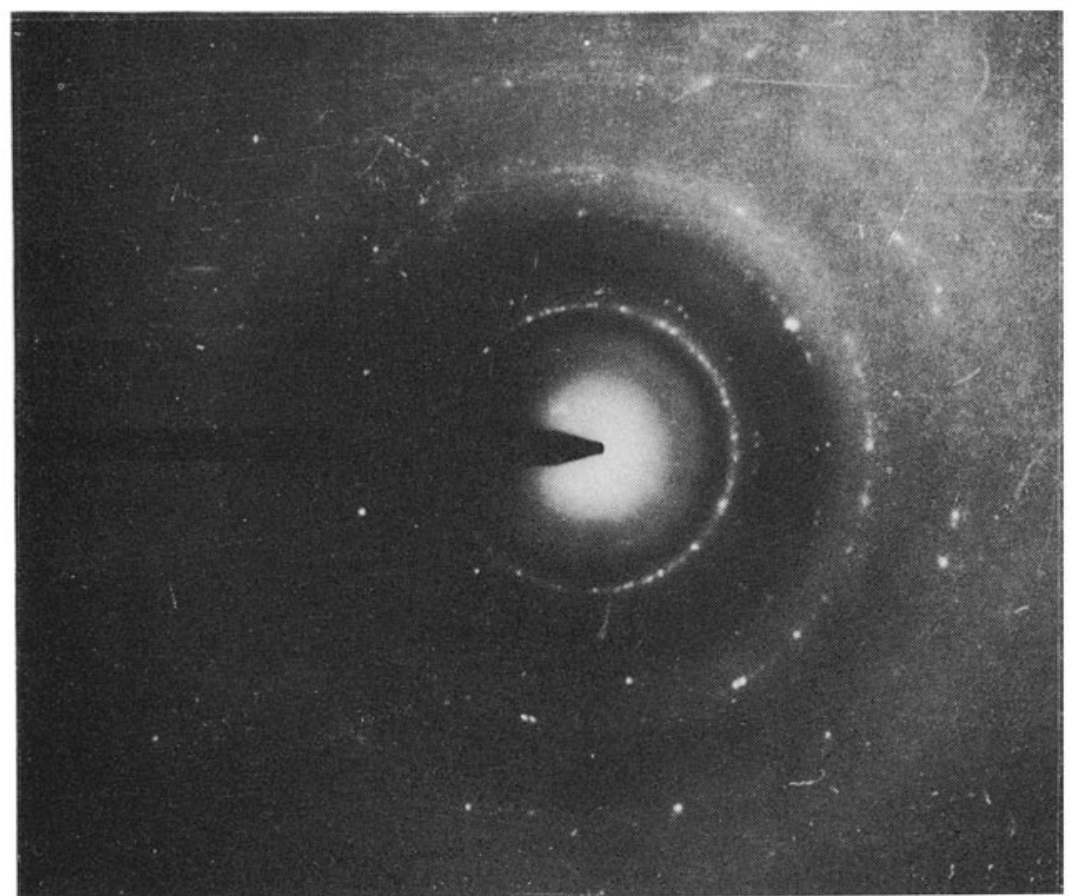

(b)

Figure 1. (a) Transmission electron micrograph for a glass of composition $80 \mathrm{~V}_{2} \mathrm{O}_{5}$ $15 \mathrm{P}_{2} \mathrm{O}_{5}-5 \mathrm{Bi}_{2} \mathrm{O}_{3}(\mathrm{~mol} \%)$ reduced at $200^{\circ} \mathrm{C}$ for $2 \mathrm{~h}$. (b) Selected area diffraction pattern for the micrograph in figure $1 \mathrm{a}$. 
Subject to constraints,

$$
\left.\begin{array}{r}
\xi \geqslant 0 \\
\xi \geqslant 0 \\
\xi+\xi \leqslant 1
\end{array}\right\}
$$

where, $\mathbf{X}$ is the vector consisting of $\xi$ and $\zeta$, the optimization variables. The above constrained optimization is converted into an equivalent unconstrained optimization by augmenting the function $f(\mathbf{X})$ by a penalty term within the frame work of interior penalty function method (Rao 1979). The equivalent function $\phi\left(\mathbf{X}, r_{k}\right)$ is given by,

$$
\phi\left(\mathbf{X}, r_{k}\right)=f(\mathbf{X})-r_{k} \sum_{j=1}^{m} \frac{1}{g_{j}(\mathbf{X})},
$$

where, $r_{k}$ is the penalty parameter and $g_{j}(\mathbf{X}) \leqslant 0$ is the constraint. In our case,

$$
\sum_{j=1}^{m} \frac{1}{g_{j}(\mathbf{X})}=\left[-\frac{1}{\xi}-\frac{1}{\zeta}+\frac{1}{\xi+\zeta-1}\right]
$$

In order to calculate $f(\bar{X})$ from (4), we have to find an expression for $\bar{\varepsilon}_{1}$ and $\bar{\varepsilon}_{2}$. Separating the real and imaginary parts in (1) we get,

$$
\begin{aligned}
& \bar{\varepsilon}_{1}=\varepsilon_{m} \frac{A_{1} C_{1}-B_{1} D_{1}}{C_{1}^{2}+D_{1}^{2}}, \\
& \bar{\varepsilon}_{2}=\varepsilon_{m} \frac{B_{1} C_{1}+A_{1} D_{1}}{C_{1}^{2}+D_{1}^{2}}
\end{aligned}
$$

where, $A_{1}, B_{1}, C_{1}$ and $D_{1}$ are given by,

$$
\begin{aligned}
& A_{1}=1+\frac{2}{3} f\left[\xi \sum_{j} \omega_{j} \delta_{j \mid \text { sphere }}^{\prime}+\zeta \delta_{\mid \text {s.c. }}^{\prime}+\chi \delta_{\mid \text {f.c. }}^{\prime}\right] \\
& B_{1}=\frac{2}{3} f\left[\xi \sum_{j} \omega_{j} \delta_{j \mid \text { sphere }}^{\prime \prime}+\zeta \delta_{\mid \text {s.c. }}^{\prime \prime}+\chi \delta_{\mid \text {ff.c. }}^{\prime \prime}\right] \\
& C_{1}=1-\frac{1}{3} f\left[\xi \sum_{j} \omega_{j} \delta_{j \mid \text { sphere }}^{\prime}+\zeta \delta_{\mid \text {s.c. }}^{\prime}+\chi \delta_{\mid \text {f.c. }}^{\prime}\right] \\
& D_{1}=\frac{1}{3} f\left[\xi \sum_{j} \omega_{j} \delta_{j \mid \text { sphere }}^{\prime \prime}+\zeta \delta_{\text {|s.c. }}^{\prime \prime}+\chi \delta_{\mid \text {f.c. }}^{\prime \prime}\right]
\end{aligned}
$$

For (10) to (13) the subscripts, sphere, s.c. and f.c. stand for sphere, single strand chain and $f(c$ clusters of the bismuth phase, respectively.

$\omega_{j}$ is the weight factor and is given by,

$$
\omega_{j}=\frac{x_{j}^{3} n_{j}}{\sum x_{j}^{3} n_{j}} \text { and } f_{j}=f \omega_{j} .
$$


$x_{j}$ and $n_{j}$ are particle size and number of particles in $j$ th class. $\delta_{j}^{\prime}$ and $\delta_{j}^{\prime \prime}$ of the above equations are obtained by separating real and imaginary parts of (2) and are given by,

$$
\begin{aligned}
& \delta_{j}^{\prime}=\frac{1}{3} \sum_{k=1}^{3} \frac{E G_{k}+F H_{k}}{G_{k}^{2}+H_{k}^{2}}, \\
& \delta_{j}^{\prime \prime}=\frac{1}{3} \sum_{k=1}^{3} \frac{F G_{k}-E H_{k}}{G_{k}^{2}+H_{k}^{2}},
\end{aligned}
$$

where,

$$
\begin{aligned}
& E=\varepsilon_{j}^{\prime}-\bar{\varepsilon}_{1}, \\
& F=\varepsilon_{j}^{\prime \prime}-\bar{\varepsilon}_{2}, \\
& G_{k}=L_{k} \varepsilon_{j}^{\prime}+\left(1-L_{k}\right) \bar{\varepsilon}_{1}, \\
& H_{k}=L_{k} \varepsilon_{j}^{\prime \prime}+\left(1-L_{k}\right) \bar{\varepsilon}_{2},
\end{aligned}
$$

where $\varepsilon_{j}^{\prime}$ and $\varepsilon_{j}^{\prime \prime}$ are the size and frequency-dependent real and imaginary parts respectively of dielectric constants of the metallic phase (Das et al 1983). For evaluating $\delta_{j}^{\prime}$ and $\delta_{j}^{\prime \prime}$ for sphere, we have taken the depolarization factors as $L_{1}=L_{2}=L_{3}=1 / 3$ (Granqvist and Hunderi 1977). We also assume that the different geometrical configurations are made of identical spheres. Therefore to calculate $\delta^{\prime}$ and $\delta^{\prime \prime}$ for s.c. and f.c. we have used an average particle size computed from the TEM photograph. The effective depolarizations factors $\left(L_{1}, L_{2}\right.$ and $\left.L_{3}\right)$ used for s.c. and f.c. configurations are respectively $0.133,0.435$ and $0.435 ; 0.0865,0.0865$ and 0.827 (Granqvist and Hunderi 1977).

To compute $\bar{\varepsilon}_{1}$ and $\bar{\varepsilon}_{2}$ from (8) and (9) we have to compute $\delta_{j}^{\prime}$ and $\delta_{j}^{\prime \prime}$ which are functions of $\bar{\varepsilon}_{1}$ and $\bar{\varepsilon}_{2}$. Therefore $\bar{\varepsilon}_{1}$ and $\bar{\varepsilon}_{2}$ have to be computed by the following iterative method. First we assume the values of $\bar{\varepsilon}_{1}$ and $\bar{\varepsilon}_{2}$ to be given by

$$
\bar{\varepsilon}_{1}^{(0)}=\varepsilon_{m} \text { and } \varepsilon_{2}^{(0)}=0 .
$$

With these values we calculate

$$
\begin{aligned}
& \bar{\varepsilon}_{1}^{(1)}=F_{1}\left(\bar{\varepsilon}_{1}^{(0)}, \bar{\varepsilon}_{2}^{(0)}\right) ; \\
& \bar{\varepsilon}_{2}^{(1)}=F_{2}\left(\bar{\varepsilon}_{1}^{(0)}, \varepsilon_{2}^{(0)}\right) .
\end{aligned}
$$

The exact functional forms of (21) and (22) are defined by (8) to (20). Here the superscript indicates the number of iterations. Substituting $\bar{\varepsilon}_{1}^{(1)}$ and $\vec{\varepsilon}_{2}^{(1)}$ in (21) and (22) we get $\bar{\varepsilon}_{1}^{(2)}$ and $\bar{\varepsilon}_{2}^{(2)}$ and so on. So in the $i$ th iteration,

$$
\begin{aligned}
& \bar{\varepsilon}_{1}^{(i)}=F_{1}\left(\bar{\varepsilon}_{1}^{(i-1)}, \bar{\varepsilon}_{2}^{(i-1)}\right), \\
& \bar{\varepsilon}_{2}^{(i)}=F_{2}\left(\bar{\varepsilon}_{1}^{(i-1)}, \bar{\varepsilon}_{2}^{(i-1)}\right),
\end{aligned}
$$

This iteration is continued until the difference between the two successive iterations is less than a pre-assigned positive quantity $e$, i.e. when simultaneously,

$$
\begin{array}{r}
\left|\bar{\varepsilon}_{1}^{(i)}-\bar{\varepsilon}_{1}^{(i-1)}\right|<e, \\
\text { and }\left|\bar{\varepsilon}_{2}^{(i)}-\bar{\varepsilon}_{2}^{(i-1)}\right|<e .
\end{array}
$$


Here in our problem we have chosen $e=0 \cdot 1$.

The computed values of $\bar{\varepsilon}_{1}$ and $\bar{\varepsilon}_{2}$ are substituted in (3) to calculate the frequency dependent $\alpha(\omega)$. This in turn is substituted in (4) to compute the value of $f(x)$.

The following iterative scheme has been used to optimize the function $f(\mathbf{X})$ subject to the constraints as mentioned earlier within an error of $\pm 4 \%$.

Step 1: The computation is started with three initial feasible points $\mathbf{X}_{1}, \mathbf{X}_{2}$ and $\mathbf{X}_{3}$ (to define the initial simplex) such that strictly the constraints $g_{j}\left(\mathbf{X}_{i}\right)<0$ for $i=1$ to 3 and $j=1$ to 3 are satisfied;

set $K \leftarrow 1$ and take $r_{k}>0$.

Step 2: $\phi\left(\mathbf{X}, r_{k}\right)$ is minimised using simplex optimization technique (Rao 1979) and the centroid of the latest simplex $\mathbf{X}_{k}^{*}$ for which convergence criterion is satisfied is taken as the optimum point.

Step 3: If $K \neq 1$, go to step 5.

Step 4: Set $F_{1} \leftarrow F\left(\mathbf{X}_{k}^{*}\right)$ and go to step 8 .

Step 5: Set $F_{2} \leftarrow F\left(\mathbf{X}^{*}\right)$

Step 6: If $\left|\frac{F_{2}-F_{1}}{F_{2}}\right| \leqslant \varepsilon$ go to step 10 .

In our problem $\varepsilon=0.04$ is assigned.

Step 7: Set $F_{1} \leftarrow F_{2}$

Step 8: Compute the new penalty parameter

$r_{k+1}=c r_{k}$, where $c<1$

Step 9: Take $\mathbf{X}_{k 1}^{*}, \mathbf{X}_{k 2}^{*}$ and $\mathbf{X}_{k 3}^{*}$ points of the latest simplex, as the starting points.

Set $K \leftarrow K+1$ and repeat step 2 .

Step 10: Take $\mathbf{X}_{k}^{*}$ as the optimum vector.

Step 11: Compute $\alpha(\omega)^{\mathrm{MG}-\mathrm{Pvs}}$ by using (3) at $\mathbf{X}_{k}^{*}$.

Step 12: Stop.

Based on the above algorithm a programme in FORTRAN language has been written (Das 1982) and run on the DEC system 1090.

\section{Discussion}

Figure 2 shows the plot of $\alpha$ values as the function of wavelength $\lambda$ for $80 \mathrm{~V}_{2} \mathrm{O}_{5}-$ $15 \mathrm{P}_{2} \mathrm{O}_{5}-5 \mathrm{Bi}_{2} \mathrm{O}_{3}(\mathrm{~mol} \%)$ glass films reduced at $200^{\circ} \mathrm{C}$ for $2 \mathrm{~h}$. The optimum combination of geometrical configurations of the metallic bismuth phase is found to be $26 \%$ sphere, $1 \%$ single strand chains and $73 \% f c c$ clusters. The fill factor and the average particle size $\bar{X}$ of the metallic phase in this composite as computed from electron micrographs are 0.033 and $10 \mathrm{~nm}$ respectively. The entire region of spectrum is not in a non-dispersive region, but the effective medium theories invoked here are not dependent on this property of the metal phase. In figure 2 are 


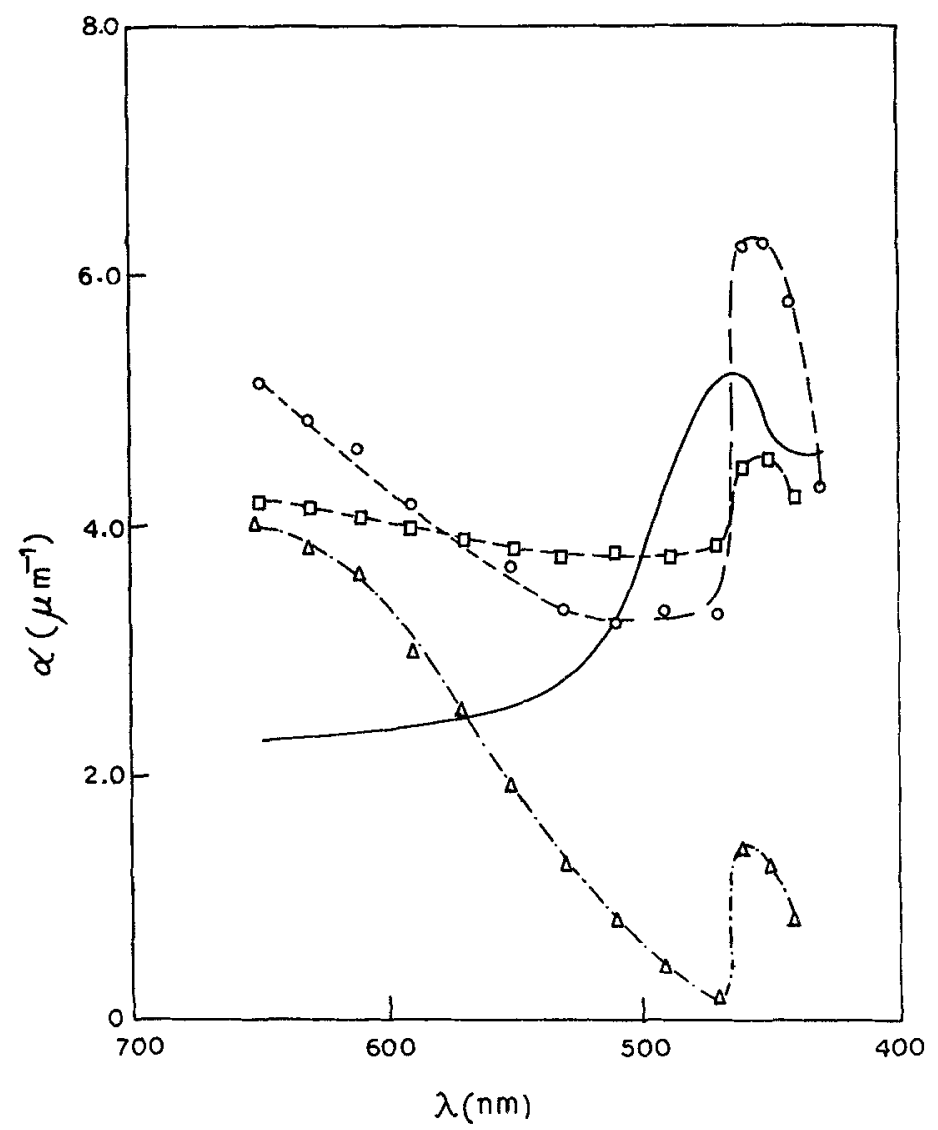

Figure 2. Comparison of experimental absorption coefficient $\alpha$ as a function of wavelength with theoretical values computed by different models for glass-metal nanocomposite thin films reduced at $200^{\circ} \mathrm{C}$ for $2 \mathrm{~h}$. Glass composition: $80 \mathrm{~V}_{2} \mathrm{O}_{5}-15 \mathrm{P}_{2} \mathrm{O}_{5}-5 \mathrm{Bi}_{2} \mathrm{O}_{3}(\mathrm{~mol} \%$ ), $f=0.033, \bar{X}=10 \mathrm{~nm}$. ( - Experimental; $O \mathrm{MG}$ theory; $\triangle \mathrm{MG}-\mathrm{PVS}$ theory; $\square$ constrained optimization.)

also shown the curves as computed by MG and MG-PVS theories, assuming the particles to consist of spheres only, and also the experimental curve for the purpose of comparison. It is evident that the curve as obtained by the optimization technique is a much better fit to the experimental data than that predicted by either the MG or the MG-PVS theory. The presence of $f(c)$ clusters and single strand chains formed by the metallic bismuth phase in the nanocomposite under investigation therefore makes the particle stretching model for memory switching (Das and Chakravorty 1980) a realistic proposition.

\section{Acknowledgement}

Financial assistance received from the Science and Engineering Research Council, Department of Science and Technology, is acknowledged. 


\section{References}

Chakravorty D and Murthy C S 1975 J. Phys. D8 L162

Craighead H G and Buhrman R A 1977 Appl. Phys. Lett. 31423

Das G C 1982 Memory switching and optical properties of glass-metal microcomposites, Ph.D. thesis, Indian Institute of Technology, Kanpur

Das G C and Chakravorty D 1980 J. Appl. Phys. 513896

Das G C and Chakravorty D 1982 J. Phys. D15 2255

Das G C, Das R and Chakravorty D 1983 Bull. Mater. Sci. 5277

Doremus R H 1964 J. Chem. Phys. 402389

Granqvist C G and Buhrman R A 1976 J. Appl. Phys. 472200

Granquist C G and Hunderi O 1977 Phys. Rev. B16 3513

Granqvist C G and Hunderi O 1980 J. Appl. Phys. 511751

Kreibig U 1974 J. Phys. F4 999

Maxwell-Garnett J C 1904 Philos. Trans. R. Soc. (London) 203385

Niklasson G A, Granqvist C G and Hunderi O 1981 Appl. Opt. 2026

Polder D and Van Santen 1946 Physica (Utrecht) 12257

Rao S S 1979 Optimization theory and practice (New Delhi: Wiley Eastern) 